\title{
Is the SIOP-2001 Classification of Renal Tumors of Childhood accurate with regard to prognosis? A problem revisited
}

\author{
Jerzy Niedzielski ${ }^{1}$ Katarzyna Taran², Wojciech Młynarski ${ }^{3}$, Anna Sitkiewicz ${ }^{4}$
}

\author{
1Department of Pediatric Surgery and Urology, University Children's Hospital No. 4 \\ Medical University of Lodz, Poland \\ 2Department of Pathology, University Children's Hospital No. 4, Medical University \\ of Lodz, Poland \\ ${ }^{3}$ Department of Pediatrics, Hematology, Oncology and Diabetology, University \\ Children's Hospital No. 4, Medical University of Lodz, Poland \\ 4Department of Pediatric Surgery and Oncology, University Children's Hospital No. 4, \\ Medical University of Lodz, Poland
}

Submitted: 31 July 2011

Accepted: 2 January 2012

Arch Med Sci 2012; 8, 4: 684-689

DOI: $10.5114 /$ aoms.2012.30292

Copyright @ 2012 Termedia \& Banach

\section{Abstract}

Introduction: The goal of this study was to analyze morbidity and mortality of Wilms' tumor based on the revised SIOP-2001 classification.

Material and methods: Sixty-four patients with unilateral Wilms' tumor, 33 girls (51.5\%) and 31 boys (48.5\%), aged 1 to 144 months (mean: 42.8 months) were treated between 1993 and 2009. All patients underwent multimodal therapy according to the SIOP protocols. The follow-up period ranged from 2 to 18 years (mean: 11.6 years).

Results: Thirty-three patients (51.6\%) had intermediate-risk, 6 (9.4\%) low-risk and 25 (39\%) high-risk tumors. Stage I disease was diagnosed in 28 (43.7\%), stage II in 19 (29.7\%), stage III in 8 (12.5\%) and stage IV in 9 patients (14.1\%). Event-free survival (EFS) in the entire group was $78.1 \%$ and OS was $92.2 \%$. The EFS in stage IV (44.4\%) was significantly lower than in stage I $(82.1 \%, p=0.04)$, stage II $(89.5 \%, p=0.02)$ and in the entire group $(78.1 \%, p=0.04)$. Sixteen complications were observed in 14 children (21.9\%); metastases in 7 cases $(10.9 \%)$, 8 relapses $(12.5 \%)$ and 5 deaths $(7.8 \%)$. Blastemal (20/24 - 83.3\%) and anaplastic $(3 / 24-12.5 \%)$ subtypes were responsible for mortality in high-risk tumors (OS $-87.5 \%)$, while poorly differentiated epithelial $(7 / 34-20.6 \%)$ and regressive $(8 / 34-23.5 \%)$ subtypes decreased OS $(94.1 \%)$ in the intermediate-risk tumors.

Conclusions: The results of our study show that epithelial and regressive subtypes were responsible for mortality in the intermediate-risk Wilms' tumors.

Key words: Wilms' tumor, revised SIOP-2001 classification, epithelial subtype, regressive subtype.

\section{Introduction}

Wilms' tumor (WT), or nephroblastoma, along with CNS tumors is the most frequent solid tumor in children, representing $8-10 \%$ of pediatric malignances $[1,2]$. The treatment of Wilms' tumor has been integrated into clinical trials since the 1970s, including in Poland [3, 4]. Opposite to the National Wilms' Tumor Study Group (NWTSG) of the USA, the European

\author{
Corresponding author: \\ Prof. Jerzy Niedzielski \\ $\mathrm{MD}, \mathrm{PhD}$ \\ Department of Pediatric \\ Surgery and Urology \\ University Children's \\ Hospital No 4 \\ 36/50 Sporna St \\ 91-738 Lodz, Poland \\ Phone: +48 426177711 \\ Fax: +48 426177705 \\ E-mail: \\ jerzy.niedzielski@umed.lodz.pl
}


International Society of Paediatric Oncology (SIOP) trials and studies mainly focused on the issue of preoperative therapy to facilitate surgery of a shrunken tumor and to treat metastases as early as possible [5]. The goal of current clinical trials is to reduce therapy for children with low-risk tumors, thereby avoiding acute and long-term toxicities. At the same time, current clinical trials seek to augment therapy for patients with high-risk Wilms' tumor, including those with bilateral, anaplastic, and recurrent favorable histology tumors [6].

However, preoperative chemotherapy alters the tumor's histological features and distribution of subtypes, and makes staging more difficult. Analyses of WT subtypes from consecutive SIOP trials have resulted in removal from or addition of certain subtypes to the risk groups of the revised SIOP-2001 working classification of renal tumors of childhood [7].

The results of statistical comparative analysis performed by the present authors in previous reports indicated that the SIOP-93 classification more precisely divided WT types according to their biology and malignant potential as compared with the SIOP-01 $[8,9]$.

We decided to revisit our material ten years after our first report in order to analyze morbidity and mortality based on the revised SIOP-2001 working classification.

\section{Material and methods}

Records of 64 consecutive patients with unilateral Wilms' tumor treated at University Children's Hospital No. 4 of the University School of Medicine in Lodz in the years 1993-2009 were reviewed. There were 33 girls (51.5\%) and 31 boys (48.5\%), aged 1 to 144 months (mean age: 42.8 months). All patients underwent multimodal therapy according to the SIOP protocols. Treatment was initiated with neoadjuvant chemotherapy (ACTD - dactinomycin, VCR - vincristine) without histological verification, diagnosis being based on clinical presentation and results of imaging studies. Surgical removal of the tumor with involved kidney was performed after initial chemotherapy. Restaging was performed and adjuvant chemotherapy was administered after histological diagnosis of the tumor was established (ACTD - dactinomycin, VCR - vincristine, EPI - epirubicin, IFO - ifosfamide, VP-16 - etoposide, CBDCA - carboplatin). Radiation therapy was applied additionally in 14 children with locoregional progression of the neoplasm (21.9\%). In 9 infants under 6 months of age (14.1\%) the treatment began with primary surgical removal of the tumor without neoadjuvant chemotherapy.

The assessment of the clinical course of the disease included: presence of distant metastases (M) detected at the diagnosis or during therapy, local recurrence of tumor confirmed by means of imag- ing studies, progression of the disease and deaths. The overall survival rate was calculated in the entire group of children with Wilms' tumor as well as in consecutive subgroups related to the clinical stage and tumor histological features.

The removed tumors were assessed histologically at the Department of Pathomorphology of University Children's Hospital No. 4 in Lodz according to the revised SIOP-2001 working classification of renal tumors of childhood. Results were verified at the Department of Pathology of the Institute of Mother and Child in Warsaw. Patients were divided into three groups: low, intermediate and highrisk tumors.

The clinical staging of tumors was done following the National Wilms' Tumor Study Group (NWTSG) in the SIOP modification [1-3]. The followup period ranged from 2 to 18 years (mean: 11.6 years). The obtained results were subjected to a statistical analysis using the Statistica 9.1 PL software package.

\section{Results}

The histology in the group of 64 children was of intermediate risk in 33 patients (51.6\%), 6 tumors (9.4\%) were low risk and 25 (39\%) high risk. The stage distribution of the tumors was: stage I in $28(43.7 \%)$, stage II in $19(29.7 \%)$, stage III in $8(12.5 \%)$ and stage IV in 9 patients (14.1\%). No predominance of either sex was observed in any of the four stage groups. Children in stage I were significantly younger ( $p=0.02)$ than patients in stage III. The average age was comparable in other stages and the entire group (Table I).

Forty-seven out of 64 children (73.4\%) were in clinical stage I and II; however, 26 of them had tumors of intermediate risk and 17 had tumors of high risk. Fifteen out of 17 patients (88.2\%) in stage III and IV had tumors of intermediate and high risk. On the other hand, 18 of 34 children (52.9\%) with intermediate-risk tumors were in stage I and 17 of 24 patients $(70.8 \%)$ with high-risk tumors were in stage I and II.

Survival analysis was performed in consecutive clinical stages in order to detect any correlation between the course of disease and clinical stage of tumor determined at the beginning of the disease following NWTSG. However, no such correlation was found in the examined group.

Event-free survival (EFS) in the entire examined group was $78.1 \%$ as compared to overall survival (OS) of $92.2 \%$. The OS of $100 \%$ was observed in all patients with low-risk tumors, in patients in stage III and IV with intermediate-risk tumors and unexpectedly in patients in stage IV with high-risk tumors. However, no significant difference was found in EFS and OS between patients with low, intermediate and high-risk tumors (Table II). 
Table I. Sex, age and number of patients regarding clinical stage of Wilms' tumor (NWTSG)

\begin{tabular}{|c|c|c|c|c|c|c|}
\hline \multirow{2}{*}{$\begin{array}{l}\text { Clinical } \\
\text { stage of } \\
\text { disease }\end{array}$} & \multicolumn{3}{|c|}{ Number of patients } & \multirow{2}{*}{$\begin{array}{c}\text { Age } \\
\text { [months] }\end{array}$} & \multirow{2}{*}{$\begin{array}{c}\text { Median } \\
\text { [months] }\end{array}$} & \multirow{2}{*}{$\begin{array}{c}\text { Mean age ( } \pm \text { SD) } \\
\text { [months] }\end{array}$} \\
\hline & M & $\mathrm{F}$ & Total, $n(\%)$ & & & \\
\hline 1 & 12 & 16 & $28(43.7)$ & $1-123$ & 28.5 & $32.5(28.93)$ \\
\hline II & 9 & 10 & $19(29.7)$ & $4-140$ & 44 & $41.47(30.95)$ \\
\hline III & 6 & 2 & $8(12.5)$ & $2-144$ & 71.5 & $66.5(51.33)$ \\
\hline IV & 4 & 5 & $9(14.1)$ & $9-124$ & 55 & $56.44(38.89)$ \\
\hline Total & 31 & 33 & 64 (100) & $1-144$ & 36 & $42.78(35.54)$ \\
\hline
\end{tabular}

$M$ - male, F-female

Table II. Event-free survival rate and overall survival rate in relation to histological type of Wilms' tumor (SIOP-01)

\begin{tabular}{|c|c|c|c|c|c|}
\hline $\begin{array}{l}\text { Histological type } \\
\text { of Wilms' tumor } \\
\text { (SIOP-01) }\end{array}$ & $\begin{array}{c}\text { No. of } \\
\text { patients }\end{array}$ & $\begin{array}{l}\text { Patients with } \\
\text { complications }\end{array}$ & Deaths & $\begin{array}{c}\text { Event-free survival } \\
\text { rate [\%] }\end{array}$ & $\begin{array}{c}\text { Overall survival } \\
\text { rate [\%] }\end{array}$ \\
\hline Low risk & 6 & 1 & None & 83.3 & 100.0 \\
\hline Intermediate risk & 34 & 5 & 2 & 85.3 & 94.1 \\
\hline High risk & 24 & 8 & 3 & 66.7 & 87.5 \\
\hline Total & 64 & 14 & 5 & 78.1 & 92.2 \\
\hline
\end{tabular}

Table III. Event-free survival rate and overall survival rate in relation to clinical stage of Wilms' tumor (NWTS)

\begin{tabular}{|cccccc|}
\hline $\begin{array}{c}\text { Clinical stage of } \\
\text { disease (NWTS) }\end{array}$ & $\begin{array}{c}\text { No. of } \\
\text { patients }\end{array}$ & $\begin{array}{c}\text { Patients with } \\
\text { complications }\end{array}$ & Deaths & $\begin{array}{c}\text { Event-free survival } \\
\text { rate [\%] }\end{array}$ & $\begin{array}{c}\text { Overall survival } \\
\text { rate [\%] }\end{array}$ \\
\hline I & 28 & 5 & 2 & 82.1 & 92.9 \\
\hline II & 19 & 2 & 1 & 75 & 89.5 \\
\hline III & 8 & 2 & None & 44.4 & 100 \\
\hline IV & 9 & 5 & 5 & 78.1 & 92.5 \\
\hline Total & 64 & 14 & & 2 & 75.5 \\
\hline
\end{tabular}

The EFS rate of children in clinical stage IV (44.4\%) was significantly lower than in stage I $(82.1 \%, p=0.04)$, stage II $(89.5 \%, p=0.02)$ and in the entire group (78.1\%, $p=0.04)$. The OS rate was highest in patients in stage $4(100 \%)$ but the difference was not significant (Table III).

Sixteen complications of neoplastic disease were observed in 14 children (21.9\%). Metastatic disease was present in 7 cases (10.9\%), in 3 patients at diagnosis and in 4 during therapy, with lungs (5/7), liver (1/7) and other (1/7) involvement. There were 8 relapses (12.5\%) and five deaths (7.8\%). The highest diversity of the course of disease was observed in children with high-risk tumors; out of 24 patients there were 5 cases of metastatic disease, 5 relapses and 3 deaths. Out of 33 patients with intermediate-risk tumors 1 metastatic case, 3 relapses, 1 renal vein thrombosis and 2 deaths were observed. The remaining 51 patients (79.7\%) completed the therapy and are free of disease with the follow-up period ranging from 2 to 18 years (mean: 11.9 years).
Regarding the histological structure of the tumor, blastemal subtype appeared to be the most common feature of high-risk histology (20/24 - 83.3\%) and together with the anaplastic subtype (3/24 $12.5 \%$ ) was responsible for mortality in this group (OS - 87.5\%). A poorly differentiated epithelial subtype $(7 / 34-20.6 \%)$ and regressive subtype $(8 / 34$ $-23.5 \%)$ were features with decreased OS (94.1\%) in the group of intermediate-risk tumors (2/34 $5.9 \%$ ) (Tables II and IV). Survival rate of children with Wilms' tumor ranged in the present study from $66.7 \%$ to $100 \%$ depending on histological subtype of the tumor. Mortality was associated with four histological subtypes: blastemal, diffuse anaplasia, low differentiated epithelial and regressive. The EFS rate of patients with blastemal WT subtype (65\%) was significantly lower than in all other subtypes not responsible for mortality in the group (92.3\%, $p=0.029)$. The EFS and OS rates of blastemal and anaplastic subtypes together were lower than those of all other subtypes ( $p=0.03, p=0.09)$. Children with epithelial and regressive subtypes together 
Table IV. Event-free survival rate and overall survival rate in relation to histological subtype of Wilms' tumor (SIOP-01)

\begin{tabular}{|lccccc|}
\hline $\begin{array}{l}\text { Histological subtype } \\
\text { of tumor (SIOP-01) }\end{array}$ & $\begin{array}{c}\text { No. of } \\
\text { patients }\end{array}$ & $\begin{array}{c}\text { Patients with } \\
\text { complications }\end{array}$ & Deaths & $\begin{array}{c}\text { Event-free (EFS) [\%] } \\
\text { survival rate }\end{array}$ & $\begin{array}{c}\text { Overall survival } \\
\text { rate (OS) [\%] }\end{array}$ \\
\hline Blastemal & 20 & 7 & 2 & 65 & 90 \\
\hline Diffuse anaplasia & 3 & 1 & 1 & 66.7 & 66.7 \\
\hline $\begin{array}{l}\text { Epithelial (poorly } \\
\text { differentiated) }\end{array}$ & 7 & 2 & 1 & 71.4 & 85.7 \\
\hline Regressive & 8 & 2 & 1 & 75 & 87.5 \\
\hline All others & 26 & 2 & None & 92.3 & 100 \\
\hline Total & 64 & 14 & 5 & 78.1 & 92.2 \\
\hline
\end{tabular}

also have EFS and OS rates lower than all other subtypes, but the difference was not significant. Further, there was no significant difference in EFS and OS rates between patients with blastemal and anaplastic subtypes together and epithelial and regressive subtypes together (Table IV).

\section{Discussion}

All trials and studies of the International Society of Paediatric Oncology (SIOP) recognized three prognostic groups of renal tumors of childhood: low-risk, intermediate-risk, and high-risk tumors, which were defined in the SIOP-1993 Working Classification of Renal Tumors of Childhood. The results of the 1993-2001 SIOP Trials showed that certain histological features of WT which remain after preoperative chemotherapy, such as blastema, are of prognostic significance while others are not. Therefore, in the present SIOP Trial and Study a revised SIOP-2001 classification of renal tumors is followed. It still recognizes the three tumor risk groups with different types in each of them, but for treatment purposes, only three major types of nephroblastoma are important: completely necrotic (low-risk tumor), blastemal (high-risk tumor), and others (intermediate-risk tumors) [5, 7].

Preoperative chemotherapy significantly alters the histological features of WT. In the earlier SIOP study, the most common subtype of the immediately operated WTs was mixed (45.1\%), followed by blastemal (39.4\%) and epithelial predominant (15.5\%), whereas in tumors that received preoperative chemotherapy, the most common type was regressive (37.6\%), followed by mixed (29.4\%), stromal (14\%), blastemal (9.3\%) and epithelial predominant (3.1\%); $6.6 \%$ of tumors were completely necrotic $[10,11]$.

Because the prognostic significance of all histological components of Wilms tumors was supposed to be studied in the present SIOP-2001 Trial we decided to analyze it in our series.

The EFS after 5 years reported by the SIOP 93-01/GPOH Trial and Study was 91\% for patients with unilateral non-metastatic WT [5]. In our series
EFS was lower (79.7\%) as compared to overall survival (OS) of $92.2 \%$. However, the series included patients with metastases, relapses and deaths.

The EFS and OS rates of patients with WT presented in this study differed in consecutive histological subtypes, being decreased in blastemal, anaplastic, epithelial, and regressive subtypes. This clearly shows that not only anaplastic and blastemal but also epithelial and regressive WT subtypes are associated with high malignancy and poor prognosis in comparison with all other subtypes, in which a fatal course of disease was not observed.

Our analysis revealed that half of all treated patients were classified as having intermediate-risk tumors and one third as having high-risk tumors. Out of 14 patients with complications of WT, 4 were classified as having intermediate and 9 as having high-risk tumors, which gives a complication rate of respectively $11.8 \%$ and $37.5 \%$. However, out of 5 deaths observed in the entire group of patients, 3 were caused by high-risk tumors, while the other 2 occurred in children with intermediate-risk tumors. This fact is not consistent with the assumption of the SIOP-2001 Trial that a fatal course of disease is associated with blastemal or diffuse anaplastic type of WT classified as high-risk tumors [5].

Unexpectedly, intermediate-risk tumors - epithelial (poorly differentiated) and regressive subtypes - were responsible for 2 deaths in our series. An interesting observation is that we found no significant difference in EFS and OS rates of patients with blastemal and anaplastic subtypes together in highrisk tumors as compared with epithelial and regressive subtypes together in intermediate-risk tumors.

The common epithelial subtype of intermediaterisk tumors of the revised SIOP-2001 classification includes both poorly differentiated and well-differentiated subtypes of the SIOP-93 classification, although their biology and prognosis differ [7]. Epithelial type is diagnosed when at least $2 / 3$ of the viable tumor consists of epithelial structure; the stromal components may comprise the rest of the viable tumor with the blastemal part comprising less than $10 \%$ of the tumor [10]. The fatal case with 
epithelial features of WT in our series was not surprising because in previous reports we observed that epithelial type of nephroblastoma shows a mixture of results typical for both intermediate- and high-risk tumors [8, 9].

Hazen-Martin et al. performed an interesting experimental study on the epithelial cell line associated with anaplastic WT. The anaplastic tumor generated mouse heterotransplants that were readily used to establish epithelial cell cultures, which, in turn, produced tumors when re-injected into nude mice. Microscopic evaluation revealed that the anaplastic epithelial cells were less differentiated than epithelial cells in classical WT. In general the molecular profile of the anaplastic tumor was more consistent with that of an epithelial-rich classic WT than with the classic triphasic WT containing blastema, stroma, and epithelial tubules. A comparison of cultures derived from an epithelial-rich, classic WT and the anaplastic WT indicated that both lacked IGF-2 and WT-1 mRNA expression. However, the well-differentiated epithelial cell culture of the classic WT expressed C-myc, Pax-8, and Pax-2 mRNA, none of which were expressed by the anaplastic epithelial cells. Furthermore, the well-differentiated epithelial cells failed to express N-myc, which was expressed by both the primary triphasic WT and the anaplastic tumor. These findings indicate that patterns of gene expression within a single component do not correlate with the aggressive clinical behavior of the tumor [12].

According to the pathological criteria of the revised SIOP-2001 classification the regressive type of WT is regarded as a tumor in which chemotherapy-induced changes comprise more than $2 / 3$ of the tumor mass. If regressive changes comprise less than $2 / 3$ of a tumor mass it is classified according to the predominant histological component (blastemal, epithelial or stromal type) $[7,10]$. In practice this means that if after pre-operative chemotherapy half of the tumor is necrotic and the other half is blastemal it will be classified as blastemal type and a high-risk tumor. However, if the tumor responded to chemotherapy and $67 \%$ of its mass is necrotic it becomes a regressive type, although the rest of the tumor is blastemal, and in consequence it is classified as intermediate-risk WT. The reason for this change is based on the results of previous SIOP trials showing that tumors with chemotherapy-resistant blastema had a worse prognosis $[5,6,13]$.

The histological picture of the other fatal case of our series classified as regressive type revealed less than $10 \%$ of viable tumor with dominant stromal feature, a little epithelial tissue and single foci of blastema. It is difficult to explain this death. Although stromal WT usually shows minimal to moderate chemotherapy-induced changes since stromal tissue seems to be resistant to chemotherapy, it should not be responsible for mortality in WT $[5,9,10]$. Preoperative chemotherapy is more likely to destroy blastema and (less differentiated) epithelial elements, while it induces maturation especially in the stromal component, where rhabdomyoblastic differentiation is much more common than in primarily operated tumors [10].

Present treatment for Wilms' tumor is very successful. Now, efforts are aimed at reducing the toxicity and burden of treatment by shortening schedules without loss of effectiveness. Shortening duration of chemotherapy could reduce acute and late side-effects and inconvenience for patient and parents while maintaining effectiveness, and could be beneficial in terms of health costs $[5,6,14]$. However, changing the pathological criteria of tumor assessment could lead to a situation in which tumors earlier classified into different subtypes and risk groups and having a different prognosis are now gathered in common regressive or mixed subtype.

In conclusion, tumor response to preoperative chemotherapy is an indicator of good prognosis [13], so completely necrotic WT has been placed in the low-risk tumor group in the current SIOP-2001 trial [7]. In the same way, the presence of a certain amount of blastema after preoperative chemotherapy clearly indicates its non-responsiveness to chemotherapy, and is associated with poorer outcome, and blastemal subtype was therefore moved into the high-risk tumor group [7]. Although preliminary results of the SIOP-2001 trial have indicated good prognosis in epithelial, stromal and regressive WT subtypes classified in the intermediate-risk group [10,15], the results of our study show that these histological patterns of WT need further investigation.

\section{Acknowledgments}

The authors thank Dr. John N. Schullinger of Morgan Stanley Children's Hospital of New York for reviewing the manuscript.

\section{References}

1. Green DM, Coppes MJ, Breslow NE, et al. Wilms tumor. In: Principles and practice in pediatric pncology. 3rd ed. Pizzo PA, Poplack DG (eds). Lippincott-Raven Publishers, Philadelphia 1997; 733-59.

2. Woźniak W. Nowotwory lite u dzieci. In: Onkologia kliniczna. Vol. 2. Krzakowski M (ed). Borgis, Warszawa 2001; 556-603.

3. D'Angio GJ, Breslow N, Beckwith JB, et al. Treatment of Wilms' tumor: results of the Third National Wilms' Tumor Study. Cancer 1989; 64: 349-59.

4. Tournade MF, Com-Hougue C, Voute PA, et al. Results of the sixth International Society of Pediatric Oncology Wilms' Tumor trial and study: a risk-adapted therapeutic approach in Wilms' tumor. J Clin Oncol 1993; 11: 1014-23. 
5. Reinhard H, Semler O, Burger D, et al. Results of the SIOP 93-01/GPOH trial and study for the treatment of patients with unilateral nonmetastatic Wilms' tumor. Klin Pediatr 2004; 216: 132-40.

6. Metzger ML, Dome JS. Current therapy for Wilms' tumor. Oncologist 2005; 10: 815-26.

7. Vujanić GM, Sandstedt B, Harms D, Kelsey A, Leuschner I, de Kraker J. SIOP Nephroblastoma Scientific Committee. Revised International Society of Paediatric Oncology (SIOP) working classification of renal tumors of childhood. Med Pediatr Oncol 2002; 38: 79-82.

8. Taran K, Sitkiewicz A, Kobos J. Histoclinical study of nephroblastoma in relation to current and previous SIOP classification of renal tumors of childhood. Pol J Pathol 2010; 4: 234-9.

9. Niedzielski J, Becht R, Taran K. Should low differentiated epithelial feature of histological structure of tumor be considered a prognostic factor in Wilms tumor? Urol Pol 2006; 59: 74-8.

10. Vujanić GM, Sandstedt B. The pathology of Wilms' tumour (nephroblastoma): the International Society of Paediatric Oncology approach. J Clin Pathol 2010; 63: 102-9.

11. Mitchell C, Pritchard Jones K, Shannon R, et al. Immediate nephrectomy versus pre-operative chemotherapy in the management of non-metastatic Wilms' tumor: results of a randomized trial (UKWT3) by the UK Children's Cancer Study Group. Eur J Cancer 2006; 42: 2554-62.

12. Hazen-Martin DJ, Re GG, Garvin AJ, Sens DA. Distinctive properties of an anaplastic Wilms' tumor and its associated epithelial cell line. Am J Pathol 1994; 144: 1023-34.

13. Boccon-Gibod L, Rey A, Sandstedt B, et al. Complete necrosis induced by preoperative chemotherapy in Wilms tumor as an indicator of low risk: report of the international society of pediatric oncology (SIOP) nephroblastoma trial and study. Med Pediatr Oncol 2000; 34: 183-90.

14. de Kraker J, Graf N, van Tinteren H, et al. Reduction of postoperative chemotherapy in children with stage I intermediate-risk and anaplastic Wilms' tumour (SIOP 93-01 trial): a randomised controlled trail. Lancet 2004; 364: 1229-35.

15. Verschuur A, Vujanic G, Bohoslavsky R, et al. Wilms' tumour of stromal or epithelial subtype have a good outcome in SIOP 93-01. Pediatr Blood Cancer 2007; 49: 421-8. 